J. Klin. Endokrinol. Stoffw. 2020 - 13:124-129 https://doi.org/10.1007/s41969-020-00110-z Online publiziert: 2 . September 2020 (c) Der/die Autor(en) 2020

\section{Christian Trummer · Verena Theiler-Schwetz · Stefan Pilz}

Klinische Abteilung für Endokrinologie und Diabetologie, Univ.-Klinik für Innere Medizin, Medizinische Universität Graz, Graz, Österreich

\title{
Subakute Thyreoiditis und akute suppurative Thyreoiditis
}

\section{Einleitung}

Sowohl die subakute Thyreoiditis (SAT, auch Thyreoiditis de Quervain oder granulomatöse Thyreoiditis) als auch die akute suppurative Thyreoiditis (AST) stellen Erkrankungen der Schilddrüse dar, bei welchen es durch eine entzündliche Zerstörung von Schilddrüsengewebe zum (temporären) Auftreten einer Thyreotoxikose kommen kann und die sich mit einem klassischen triphasischen Verlauf präsentieren können (Thyreotoxikose, Hypothyreose, Genesung) [1] Während die SAT verhältnismäßig häufig beobachtet werden kann, stellt die AST eine seltene, aber möglicherweise lebensbedrohliche Erkrankung dar [2]. Da die AST eine bakterielle Infektion der Schilddrüse bezeichnet und die SAT einen abakteriellen, vermutlich viralen oder postviralen pathogenetischen Mechanismus aufweist, unterscheiden sich die Therapiemöglichkeiten drastisch [2, 3]. In diesem Artikel sollen die Pathogenese, die Diagnostik sowie die Therapie der beiden Erkrankungen dargestellt werden und insbesondere auf die klinisch wichtige Unterscheidung eingegangen werden.

\section{Subakute Thyreoiditis (Thyreoiditis de Quervain, granulomatöse Thyreoiditis)}

Die SAT wurde erstmalig im Jahr 1902 von de Quervain als eigenständiges Erkrankungsbild beschrieben und ist die häufigste granulomatöse Erkrankung der Schilddrüse [3, 4]. Sie tritt mit einer Inzidenz von ungefähr 12,1 Fällen pro 100.000 Personen pro Jahr auf und betrifft häufiger Frauen als Männer sowie
Personen im jüngeren und mittleren Erwachsenenalter $[5,6]$.

Die genaue Ätiologie der SAT ist nicht bekannt, man geht aber von einer viralen oder postviralen Genese aus, wobei Mumps, Hepatitis B und C, CMV, Enteroviren und Coxsackie-A- und -B-Viren als mögliche Auslöser diskutiert werden [7]. $\mathrm{Zu}$ dieser Annahme würde auch die höhere Inzidenz der SAT in den Sommermonaten passen, welche sich z. B. mit den Infektionsgipfeln der Coxsackie-A- und -B-Viren deckt, sowie das Auftreten von SAT-Clustern während des gleichzeitig gehäuften Auftretens von viralen Infektionen $[8,9]$. Für eine (post-)virale Genese spricht auch, dass die SAT in vielen Fällen nach Infekten der oberen Atemwege auftritt, wobei die Latenz üblicherweise zwischen 2 und 8 Wochen beträgt [10]. In der Pathogenese der SAT dürfte außerdem auch eine gewisse genetische Prädisposition eine Rolle spielen, da in bis zu $70 \%$ der Fälle eine Assoziation mit HLAB35 besteht [11]. Durch die entstehende Inflammation kommt es zu einer Beschädigung der Schilddrüsenfollikel sowie zu einer Proteolyse des in den Schilddrüsenfollikeln gespeicherten Thyreoglobulins. Wie bei anderen Formen der destruktiven Thyreoiditis werden dabei große Mengen an Schilddrüsenhormonen unkontrolliert in den Kreislauf freigesetzt, wodurch es zu einer biochemischen und klinischen Hyperthyreose kommt [12]. Im Gegensatz zu anderen Ursachen einer Hyperthyreose wie z. B. dem Morbus Basedow ist die thyreotoxische Phase bei destruktiven Thyreoiditiden - und somit auch bei der SAT - zeitlich begrenzt. Diese endet üblicherweise nach 3-6 Wochen, wenn die Speicher der bereits gebildeten Schilddrüsenhormone erschöpft sind [1].
Oft kann ein typischer triphasischer Verlauf beobachtet werden, wobei nach der initialen Hyperthyreose eine Phase der Hypothyreose aufgrund der Zerstörung von Schilddrüsenfollikeln folgt, welche üblicherweise einige Wochen bis $\mathrm{zu} 6$ Monaten dauern kann. Schließlich entwickelt der Großteil der PatientInnen nach 6-12 Monaten wieder eine normale Schilddrüsenfunktion [13]. Dennoch kann es bei bis zu einem Viertel der Betroffenen zu einer permanenten Hypothyreose kommen, die eine Schilddrüsenhormonsubstitution mit Levothyroxin erfordert [14].

Die Diagnose einer SAT wird klinisch gestellt. Schmerzen im vorderen Halsbereich in Verbindung mit einem durchgemachten Infekt der oberen Atemwege sollten an die Erkrankung denken lassen [15]. Als Kardinalsymptom der SAT tritt der Halsschmerz bei quasi allen betroffenen PatientInnen in Erscheinung [6]. Dieser kann akut oder schleichend eintreten und auf die Schilddrüsenregion begrenzt sein oder auch in den Kiefer, die Brust, den oberen Halsbereich oder den Rachen ausstrahlen $[6,16]$. Auch Fieber, Myalgien, Abgeschlagenheit oder ein schweres allgemeines Krankheitsgefühl können auftreten [16]. Laborchemisch findet sich bei vielen PatientInnen initial der biochemische Nachweis einer Hyperthyreose mit erhöhtem freiem Thyroxin (fT4) und freiem Trijodthyronin (fT3) sowie supprimiertem Thyroidea-stimulierendem Hormon (TSH) [5]. Üblicherweise fällt nur eine milde Erhöhung von fT4 und fT3 auf, während auch die beim Morbus Basedow typische disproportionale Erhöhung von fT3 fehlt [17]. Entsprechend ist die fT3/fT4-Ratio bei der SAT im Vergleich zum Mor- 


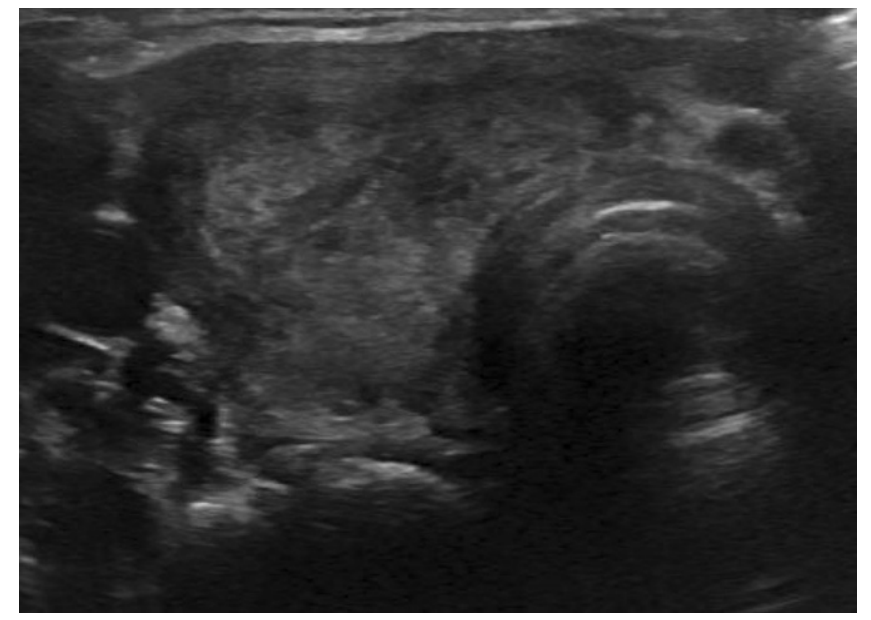

Abb. $1<$ Subakute Thyreoiditis (Querschnitt) mit typischen hypoechogenen landkartenförmigen Arealen

bus Basedow deutlich erniedrigt, typisch ist z. B. ein Wert $<0,4$ (wenn beide freien Schilddrüsenhormone in $\mathrm{pmol} / \mathrm{L}$ gemessen werden) [18]. Eine Hyperthyreose kann aber auch zu Beginn der Erkrankung (noch) nicht vorhanden sein, bzw. sehr milde Formen können auch lange euthyreot verlaufen, weshalb eine SAT bei bestehender Euthyreose nicht ausgeschlossen werden kann. Die Blutsenkungsgeschwindigkeit (BSG) beträgt meist mehr als $50 \mathrm{~mm} / \mathrm{h}$, während auch eine Leukozytose, eine milde Anämie sowie eine Erhöhung des C-reaktiven Proteins (CRP) vorkommen können [1, 15, 19]. Im Sinne der destruktiven Thyreoiditis und der damit verbundenen Zerstörung von Schilddrüsenfollikeln ist oft auch das Thyreoglobulin im Serum erhöht [12]. Typischerweise kommt es auch zu einer Erhöhung der Leberparameter, die sich im Verlauf der Erkrankung zumeist selbstständig bessert [20]. Dahingegen sind die Antikörper gegen Thyreoglobulin (Tg-Antikörper) und Thyreoperoxidase (TPO-Antikörper) bei Diagnosestellung meist nicht erhöht, sie können aber bei Auftreten der oft transienten hypothyreoten Phase vermutlich als Reaktion auf die Freisetzung von Schilddrüsenantigenen nachweisbar sein [13, 21].

Die Schilddrüsensonographie kann als einfache und schnelle Untersuchung wichtige Hinweise für die Diagnosestellung liefern. Das typische Bild der SAT umfasst fokale, schlecht abgrenzbare hypoechogene Areale, die oft „landkartenartig“ aussehen (d.h. die Areale stellen sich wie Ländergrenzen auf einer
Landkarte dar) (• Abb. 1). Die Veränderungen treten meist bilateral auf, sie können aber auch nur in einem der beiden Schilddrüsenlappen vorkommen [22]. In manchen Fällen können auch knotige Veränderungen auftreten, die teilweise mit malignitätsverdächtigen Schilddrüsenknoten verwechselt werden können (cave: anaplastisches Schilddrüsenkarzinom als seltene Alternativdiagnose) [23]. Das Schilddrüsenvolumen ist üblicherweise normal oder etwas erhöht [24]. Dopplersonographisch stellt sich die Schilddrüsendurchblutung trotz biochemischer Hyperthyreose erniedrigt dar, während sie bei Morbus Basedow klassischerweise signifikant erhöht ist [22]. In den meisten Fällen kommt es im Verlauf der Erkrankung zu einer vollständigen Rückbildung der sonographischen Veränderungen, während in manchen Fällen einzelne Residuen der lokalen hypoechogenen Areale bestehen bleiben können [23]. Szintigraphische Untersuchungen mit Radiojod oder Technetium zeigen typischerweise einen niedrigen Tracer-Uptake, insbesondere im Vergleich zum generell gesteigerten Uptake bei Morbus Basedow oder der fokal konzentrierten Aufnahme bei Schilddrüsenautonomie [15]. Prinzipiell spielt die Feinnadelpunktion der Schilddrüse bei der Diagnose der SAT eine untergeordnete Rolle, insbesondere falls sich in der Zusammenschau der klinischen und laborchemischen Befunde ein typisches Bild ergibt. Gelegentlich kann die Abgrenzung zu einem malignitätsverdächtigen Schilddrüsenknoten bzw. einer Infiltration der Schilddrüse durch einen malignen Prozess oder auch zu einer suppurativen Thyreoiditis schwierig sein. In solchen Fällen sollte die Indikation zu einer Feinnadelpunktion als ambulantes, einfaches und risikoarmes Verfahren sehr großzügig gestellt werden [25]. Aufgrund der möglichen Verwechslung mit malignen Prozessen wird von einigen Autoren bei der Diagnosestellung eine verpflichtende Feinnadelpunktion zum Ausschluss solcher Geschehen gefordert [26]. Das typische zytologische Bild nach Feinnadelpunktion einer SAT zeigt granulomatöse Infiltrate sowie Riesenzellen wie bei einer viralen Infektion [1].

Die Therapie der SAT soll zum einen $\mathrm{zu}$ einer Verbesserung von möglichen klinischen Symptomen einer Thyreotoxikose und zum anderen $\mathrm{zu}$ einer Schmerzlinderung führen. Zur Kontrolle hyperthyreoter Beschwerden wie Tachykardien oder Tremor werden wie bei anderen Formen der Schilddrüsenüberfunktion Betablocker verwendet (z. B. Propranolol) [15]. Als analgetische Erstlinientherapie werden nichtsteroidale Antirheumatika (NSAR) verwendet [12]. Gemäß verfügbaren Guidelines [1] wird bei Versagen der Therapie mit NSAR eine Behandlung mit Glukokortikoiden empfohlen. Eine fehlende Besserung unter NSAR wurde in bis zu $50 \%$ der PatientInnen beobachtet, während die Glukokortikoidtherapie insbesondere auch in der Symptomkontrolle überlegen zu sein scheint $[27,28]$, Glukokortikoide sind außerdem möglicherweise mit einem kürzeren Krankheitsverlauf assoziiert [29]. Aus diesem Grund kann je nach individuellem Fall auch gleich zu Beginn eine Glukokortikoidtherapie gestartet werden. Die Standardempfehlung ist üblicherweise $40 \mathrm{mg}$ Prednison über 1-2 Wochen, gefolgt von einem Tapering über 2-4 Wochen bzw. je nach klinischem Verlauf auch über längere Zeit [1]. Auch andere Therapieregime mit einer initialen Dosis von 25-60 mg Prednison täglich wurden erfolgreich verwendet [30], sodass die optimale Glukokortikoiddosis für die Behandlung der SAT derzeit noch unklar ist. Da jedoch sowohl die Therapie mit NSAR als auch mit Glukokortikoiden mit möglichen unerwünschten Wirkungen behaftet sind 


\section{Trummer $\cdot$ V. Theiler-Schwetz · S. Pilz \\ Subakute Thyreoiditis und akute suppurative Thyreoiditis}

\section{Zusammenfassung}

Die subakute Thyreoiditis (SAT) und die akute suppurative Thyreoiditis (AST) sind Schilddrüsenerkrankungen, bei welchen es durch Zerstörung von Schilddrüsengewebe zum Auftreten einer Hyperthyreose kommen kann. Die SAT ist vermutlich (post-)viraler Genese. Typischerweise folgt einer initial hyperthyreoten Phase oft eine zumeist transiente Hypothyreose, klinisch sind Halsschmerzen, Fieber oder Abgeschlagenheit charakteristisch. Laborchemisch werden oft erhöhte Entzündungsparameter gefunden, hilfreich für die Diagnosestellung sind außerdem ein erhöhtes Thyreoglobulin sowie hohe Leberparameter. Sonographisch zeigen sich häufig fokale, schlecht abgrenzbare hypoechogene Areale bei verminderter Schilddrüsendurchblutung, in szintigraphischen
Untersuchungen ist der Tracer-Uptake reduziert. Therapeutisch kommen Betablocker zur Linderung hyperthyreoter Beschwerden zum Einsatz, zur analgetischen Therapie werden nichtsteroidale Antirheumatika (NSAR) und Glukokortikoide eingesetzt. In schweren Fällen kann auch eine Thyreoidektomie notwendig sein, bei unklaren Fällen sollte die Indikation zu einer Feinnadelpunktion großzügig gestellt werden, um eine Abgrenzung zu malignen Prozessen oder einer AST zu ermöglichen. Im Gegensatz zur SAT handelt es sich bei der AST um eine bakterielle Infektion der Schilddrüse, die oft auf dem Boden von anatomisch-strukturellen Veränderungen entsteht. Typische Symptome umfassten eine Schwellung des Halses verbunden mit Schmerzen, Fieber, Heiserkeit, Dysphagie sowie einem lokalen Erythem. Laborchemisch zeigen sich auch hier erhöhte Entzündungsparameter, während eine Hyperthyreose seltener ist. In der akuten Entzündungsphase kann eine Abszessformation und ihre Ausdehnung mittels Ultraschall oder CT dargestellt werden. Hinsichtlich der Therapie sind eine rasche antibiotische Therapie und eine Drainage des Abszesses von großer Bedeutung, teilweise kann auch eine operative Entfernung von Schilddrüsengewebe notwendig werden.

\section{Schlüsselwörter}

Subakute Thyreoiditis - Thyreoiditis de Quervain · Granulomatöse Thyreoiditis . Akute suppurative Thyreoiditis - Destruktive Thyreoiditis

\section{Subacute thyroiditis and acute suppurative thyroiditis}

\section{Abstract}

Both subacute thyroiditis (SAT) and acute suppurative thyroiditis (AST) represent thyroid disorders that may lead to hyperthyroidism as a result of destructive thyroiditis. SAT is supposedly of (post-)viral origin and has a characteristic triphasic clinical course (hyperthyroidism, hypothyroidism, euthyroidism). Patients often suffer from local pain, fever or malaise, biochemical findings include elevated inflammation parameters and increased serum thyroglobulin and liver enzymes. Usually, focal or multifocal poorly defined hypoechoic areas and a decreased vascularity in Doppler studies can be found during ultrasound imaging, while tracer uptake is reduced in thyroid scintigraphy. Beta-blocking agents are used for symptom control during thyrotoxicosis, nonsteroidal anti-inflammatory drugs (NSAIDs) and glucocorticoids are recommended for pain relief and control of inflammatory symptoms. Thyroidectomy may be necessary in severe cases. Furthermore, unclear cases of SAT should prompt liberal further investigation via fine needle aspiration to differentiate the disease from possible malignancies or AST. AST is a bacterial infection of the thyroid, whose development is supported by certain anatomical-structural predispositions. Clinically, patients experience sudden neck swelling, pain, fever, sore throat, dysphagia, and localized erythema. SAT also leads to increased inflammatory parameters, while a thyrotoxic phase is less common. During acute inflammation, abscess formation and its expansion can be demonstrated by thyroid ultrasound or neck CT scans. Therapeutically, immediate empiric antibiotic therapy as well as drainage of abscess formations is of utmost importance.

\section{Keywords}

Subacute thyroiditis - De Quervain's thyroiditis . Granulomatous thyroiditis - Acute suppurative thyroiditis - Destructive thyroiditis bzw. bei manchen PatientInnengruppen kontraindiziert sind oder in Wechselwirkung mit anderen Medikamenten treten können, muss in jedem Fall eine auf die Patientin/den Patienten abgestimmte Therapieentscheidung getroffen werden. Wir möchten außerdem darauf hinweisen, dass es bei einer SAT insbesondere unter Therapie mit Glukokortikoiden normalerweise $\mathrm{zu}$ einer raschen Beschwerdebesserung innerhalb weniger Tage (oft schon innerhalb der ersten 24h) kommt [1, 13]. Eine fehlende Besserung unter Therapie sollte immer zu einer Reevaluierung der Diagnose einer SAT führen, wobei in solchen unklaren Fällen unserer Meinung nach auch großzügig eine Feinnadelpunktion der Schilddrüse erfolgen soll (um z.B. einen malignen Prozess ausschließen zu können). Da die Schilddrüsenhormonsynthese bei der SAT nicht gestört bzw. durch die Freisetzung von Schilddrüsenhormonen aus den beschädigten Schilddrüsenfollikeln sogar gehemmt ist, hat eine thyreostatische Therapie bei der SAT keine Bedeutung und sollte nicht verwendet werden [1]. Aufgrund ihrer milden Ausprägung wird in der oft transienten hypothyreoten Phase der SAT keine Schilddrüsenhormonsubstitution benötigt [12]. Bei Bedarf (ausgeprägte Hypothyreose, stark symptomatische PatientInnen) kann jedoch eine Standardtherapie mit Levothyroxin erfolgen, die zumeist nach 3-6 Monaten unter Kontrolle der Schilddrüsenfunktion wieder abgesetzt werden kann (in den seltenen Fällen einer permanenten Hypothyreose nach SAT muss natürlich bei diesen PatientInnen eine dauerhafte Therapie in Erwägung gezogen werden) 


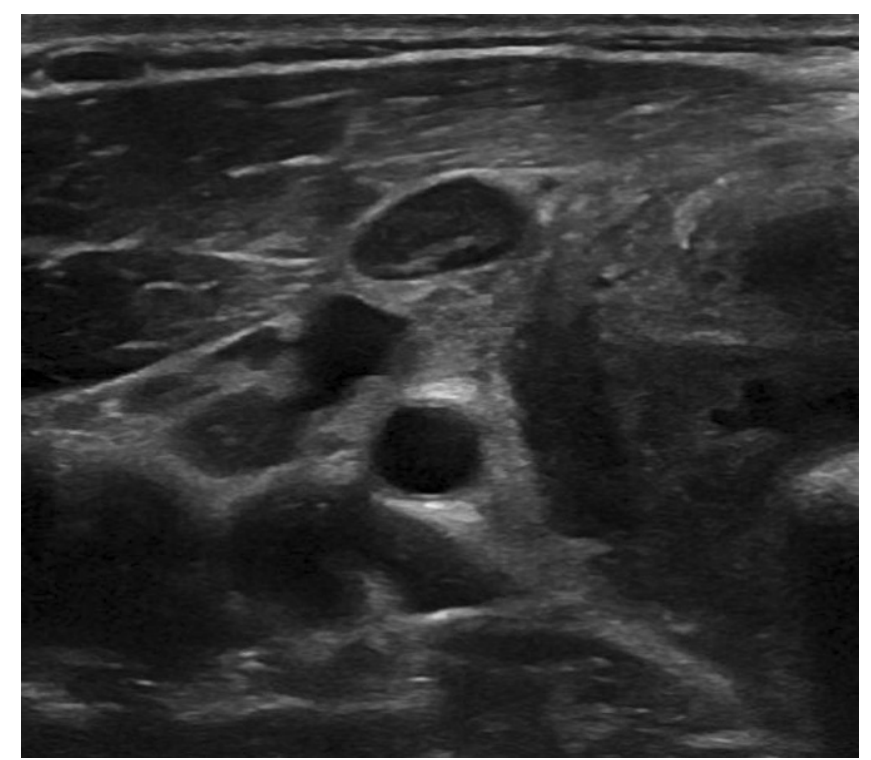

Abb. 2 \ Akute suppurative Thyreoiditis (Querschnitt rechter Schilddrüsenlappen) mit auffällig vergrößertem Lymphknoten im Bereich der Halsgefäße

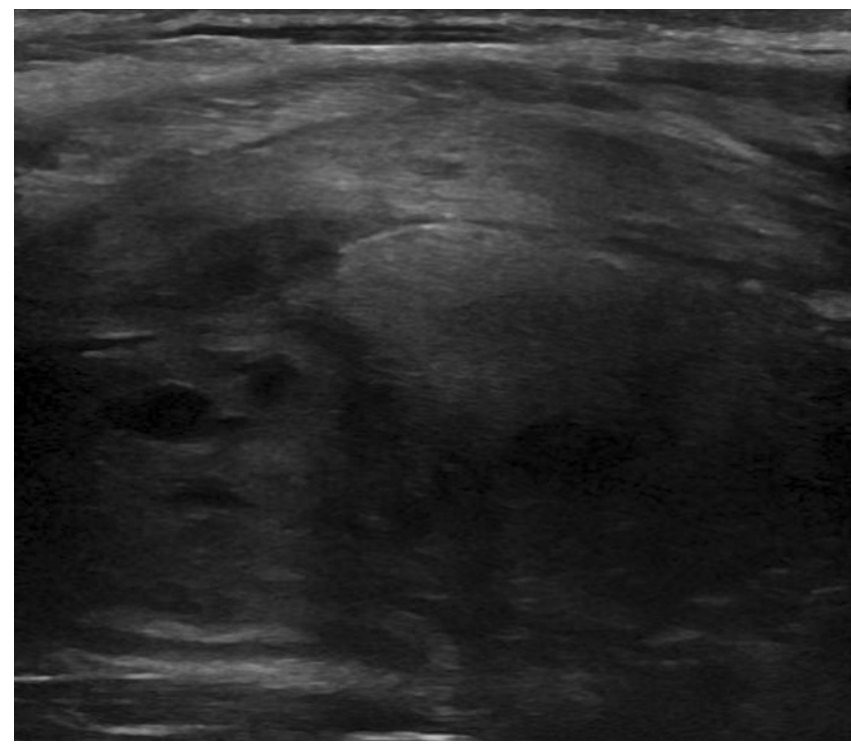

Abb. $3 \Delta$ Akute suppurative Thyreoiditis (Längsschnitt rechter Schilddrüsenlappen)
[1]. In schweren Fällen, bei welchen es trotz Glukokortikoidtherapie zu keiner Besserung der Klinik und/oder der laborchemischen Parameter kommt, bzw. bei häufigem Rückfall nach Beenden der Therapie kann auch eine Thyreoidektomie als definitive Therapie der SAT in Erwägung gezogen werden [31]. Zu einem Rezidiv kommt es in ca. $2 \%$ der Fälle [5].

\section{Akute suppurative Thyreoiditis}

Die AST stellt eine seltene, jedoch möglicherweise lebensbedrohliche Erkrankung der Schilddrüse dar [2], deren Inzidenz in der Literatur mit $0,1-0,7 \%$ aller Schilddrüsenerkrankungen angegeben wird [32]. Dies wird unter anderem dadurch erklärt, dass die Schilddrüse ein abgekapseltes Organ mit ausreichender Versorgung durch Blut- und Lymphgefäße darstellt, das außerdem durch die hohe intrathyreoidale Jodkonzentration gegenüber Infekten relativ unanfällig ist [33]. Den pathogenetischen Hintergrund der AST stellt eine bakterielle Infektion der Schilddrüse mit einem breiten möglichen Keimspektrum dar, wobei der Großteil der Fälle durch grampositive oder gramnegative Aerobier ausgelöst wird [2]. Das Risiko einer Infektion wird maßgeblich durch das Vorliegen von anatomischen Varianten oder strukturellen
Veränderungen im Bereich der Schilddrüse begünstigt (z. B. Sinus-piriformisFisteln, Traumata, operative Eingriffe im Halsbereich, etc.), eine solche Prädisposition kommt in über $70 \%$ der Fälle vor [34]. In fast einem Viertel der Fälle liegt außerdem eine vorbestehende Beeinträchtigung der Immunreaktion vor (z.B. HIV-Infektion) [34]. Im Gegensatz zur SAT tritt die AST bei Frauen und Männern annähernd gleich häufig auf [35].

Die AST präsentiert sich klinisch durch eine Schwellung des Halses verbunden mit Schmerzen, Fieber, Heiserkeit, Dysphagie sowie einem lokalen Erythem $[2,34]$. Typischerweise ist der linke Schilddrüsenlappen häufiger betroffen als der rechte $[36,37]$. Charakteristisch sind eine erhöhte BSG sowie ein erhöhtes CRP, wobei beide Marker keine bedeutende Spezifität für die AST besitzen [2]. Auch die Messung von Thyreoglobulin kann zwar Hinweise für die Schilddrüse als Ort der Inflammation geben, der Thyreoglobulinspiegel im Serum ist aber auch z. B. bei anderen Erkrankungen mit Beschädigung der Schilddrüsenfollikel erhöht [38]. Wie bei allen Formen der destruktiven Thyreoiditis kann es auch bei der AST zum Auftreten einer Thyreotoxikose kommen, diese tritt jedoch deutlich seltener auf als bei der SAT (z. B. hatten in einem Review von 191 Fällen
$83,1 \%$ eine normale Schilddrüsenfunktion) [34]. In der akuten inflammatorischen Phase zeigt sich sonographisch eine hypoechogene Läsion im und um den betroffenen Schilddrüsenlappen in Verbindung mit einer Zerstörung des Schilddrüsengewebes und einer begleitenden Abszessformation (• Abb. 2 und 3) [39]. Üblich sind auch ein hypoechogener Saum um die Schilddrüse, eine unscharfe Begrenzung zwischen der Schilddrüse und dem umliegenden Gewebe sowie ein unilaterales Auftreten [39]. Die Aufnahme von Radiojod oder Technetium ist wie bei der SAT erniedrigt [2]. Für die Akutdiagnostik ist insbesondere ein CT der Halsregion hilfreich, da dadurch auch die beteiligten anatomischen Strukturen sowie etwaige anatomische Veränderungen dargestellt werden können [39]. Außerdem kann eine mögliche Ausdehnung der Inflammation in den retropharyngealen Raum oder in das Mediastinum dargestellt werden $[40,41]$.

Aufgrund des möglicherweise dramatischen Verlaufs muss die AST von der deutlich häufigeren SAT abgegrenzt werden. Während beide Erkrankungen erhöhte Entzündungsparameter (BSG, CRP, teilweise Leukozytose) sowie einen verminderten Uptake von Radiojod/ Technetium aufweisen und prinzipiell beide Erkrankungen mit einer Thyreoto- 
xikose einhergehen können, eignen sich vor allem der Schilddrüsenultraschall und die Feinnadelpunktion zur Differenzierung [2]. Daneben können auch das unilaterale (linksseitige) Auftreten oder ein Hauterythem für das Vorliegen einer AST sprechen. Für eine SAT spricht hingegen ein Anstieg der Leberparameter sowie das rasche Ansprechen auf Glukokortikoide [15]. Selten können auch schnell wachsende Schilddrüsenkarzinome (z.B. anaplastische oder medulläre Schilddrüsenkarzinome) $\mathrm{zu}$ lokalen Infekten oder Nekrosen führen und somit das Bild einer AST imitieren. Deshalb sollte auch hier bei unklaren Fällen die Schwelle für das Durchführen einer Feinnadelpunktion zur weiteren Abklärung niedrig gehalten werden [2]. Da gemäß bestehender Fallberichte gerade in der frühen Phase einer AST nicht selten fälschlicherweise eine SAT diagnostiziert und mit Glukokortikoiden behandelt wird, möchten wir explizit darauf hinweisen, dass sich auch eine AST passager unter Glukokortikoidtherapie bessern kann und man somit bei Vorliegen einer klinischen Besserung unter Glukokortikoiden eine AST nicht ausschließen kann [42].

Aufgrund der zu erwartenden erhöhten Mortalität bei AST sollte bei quasi allen PatientInnen umgehend mit einer empirischen antibiotischen Therapie begonnen werden, nachdem Blutkulturen abgenommen wurden. Bei immunkompetenten Personen kann diese z. B. aus einem Penicillase-resistenten Penicillin in Kombination mit einem $\beta$-LaktamaseInhibitor (wie Piperacillin/Tazobactam) bestehen, bei immunkompromittierten PatientInnen müssen auch opportunistische Erreger bedacht werden und somit ein breiteres Keimspektrum abgedeckt werden [2]. Im Anschluss sollte auch invasiv Material zur weiteren kulturellen Testung gewonnen werden (Abszessflüssigkeit bzw. Gewebe) [2]. Falls es trotz antibiotischer Therapie und ggf. ultraschallgezielter Abszessdrainage via Feinnadelaspiration zu einer klinischen Verschlechterung oder zu einer Persistenz bzw. Progression der Abszessformation kommt, ist eine chirurgische Drainage oder in schweren Fällen eine totale/ subtotale Thyreoidektomie oder eine $\mathrm{He}$ - mithyreoidektomie notwendig [2, 43]. Jedenfalls sollte mit der Entscheidung zur operativen Intervention nicht zu lange zugewartet werden und die Indikation großzügig gestellt werden, da bei ausbleibender Abszessdrainage eine Dissektion in übrige Halsregionen oder den Thorax sowie eine Ruptur in die Trachea oder den Ösophagus möglich sind [44]. Auch bei der AST ist durch die destruktive Thyreoiditis eine permanente Hypothyreose mit der Notwendigkeit einer Schilddrüsenhormonsubstitution möglich [45].

\section{Korrespondenzadresse}

PD DDr. Christian Trummer

Klinische Abteilung für Endokrinologie und Diabetologie, Univ.-Klinik für Innere Medizin, Medizinische Universität Graz Auenbruggerplatz 15, 8036 Graz, Österreich christian.trummer@medunigraz.at

\section{Assoz. Prof. PD Dr. Stefan Pilz, PhD}

Klinische Abteilung für Endokrinologie und Diabetologie, Univ.-Klinik für Innere Medizin, Medizinische Universität Graz Auenbruggerplatz 15, $8036 \mathrm{Graz}$, Österreich stefan.pilz@chello.at

Funding. Open access funding provided by Medical University of Graz.

\section{Einhaltung ethischer Richtlinien}

Interessenkonflikt. C. Trummer, V. Theiler-Schwetz und S. Pilz geben an, dass kein Interessenkonflikt besteht.

Für diesen Beitrag wurden von den Autoren keine Studien an Menschen oder Tieren durchgeführt. Für die aufgeführten Studien gelten die jeweils dort angegebenen ethischen Richtlinien. Die Schilddrüsensonographiebilder entstammen den Daten der "Graz Endokrinologie Registerstudie“ und wir danken allen TeilnehmerInnen dieser Studie.

Open Access. Dieser Artikel wird unter der Creative Commons Namensnennung 4.0 International Lizenz veröffentlicht, welche die Nutzung, Vervielfältigung, Bearbeitung, Verbreitung und Wiedergabe in jeglichem Medium und Format erlaubt, sofern Sie den/die ursprünglichen Autor(en) und die Quelle ordnungsgemäß nennen, einen Link zur Creative Commons Lizenz beifügen und angeben, ob Änderungen vorgenommen wurden.

Die in diesem Artikel enthaltenen Bilder und sonstiges Drittmaterial unterliegen ebenfalls der genannten Creative Commons Lizenz, sofern sich aus der Abbildungslegende nichts anderes ergibt. Sofern das betreffende Material nicht unter der genannten Creative Commons Lizenz steht und die betreffende Handlung nicht nach gesetzlichen Vorschriften erlaubt ist, ist für die oben aufgeführten Weiterverwendungen des Materials die Einwilligung des jeweiligen Rechteinhabers einzuholen.

Weitere Details zur Lizenz entnehmen Sie bitte der Lizenzinformation auf http://creativecommons.org/ licenses/by/4.0/deed.de.

\section{Literatur}

1. Ross DS, Burch HB, Cooper DS, Greenlee MC, Laurberg P, Maia AL, Rivkees SA, Samuels $M$, Sosa JA, Stan MN, Walter MA (2016) 2016 American Thyroid Association guidelines for the diagnosis and management of hyperthyroidism and other causes of thyrotoxicosis. Thyroid 26:1343-142

2. Paes JE, Burman KD, Cohen J, Franklyn J, McHenry CR, Shoham S, Kloos RT (2010) Acute bacterial suppurative thyroiditis: a clinical review and expert opinion. Thyroid 20:247-255

3. Trivedi DP, Bhagat R, Nakanishi Y, Wang A, MorozK, Falk NK (2017) Granulomatous thyroiditis: a case report and literature review. Ann Clin Lab Sci 47:620-624

4. de Quervain F (1902) Ueber acute, nicht eiterige Thyreoiditis. Arch Klin Chir 67:706-714

5. Fatourechi V, Aniszewski JP, Fatourechi GZ, Atkinson EJ, Jacobsen SJ (2003) Clinical features and outcome of subacute thyroiditis in an incidence cohort: Olmsted County, Minnesota, study. JClin Endocrinol Metab 88:2100-2105

6. Golden SH, Robinson KA, Salanha I, Anton B, Ladenson PW (2009) Clinical review: prevalence and incidence of endocrine and metabolic disorders in the United States: a comprehensive review. JClin Endocrinol Metab 94:1853-1878

7. Alfadda AA, Sallam RM, Elawad GE, Aldhukair $H$, Alyahya MM (2014) Subacute thyroiditis: clinical presentation and long term outcome. Int J Endocrinol 2014:794943

8. Martino E, Buratti L, Bartalena L, Mariotti S, Cupini C, Aghini-Lombardi F, Pinchera A (1987) High prevalence of subacute thyroiditis during summer season in Italy. J Endocrinol Invest 10:321-323

9. Volpé R (1975) Thyroiditis: current views of pathogenesis. Med Clin North Am 59:1163-1175

10. Desailloud R, Hober D (2009) Viruses and thyroiditis: an update. Virol $\mathrm{J}$ 6:5

11. Stasiak M, Tymoniuk B, Michalak R, Stasiak B, Ko walski ML, Lewiński A (2020) Subacute thyroiditis is associated with HLA-B*18:01, $-\mathrm{DRB} 1{ }^{*} 01$ and $-C^{*} 04: 01$ - the significance of the new molecular background. JClin Med 9:534

12. Sweeney LB, Stewart C, Gaitonde DY (2014) Thyroiditis: an integrated approach. Am Fam Physician 90:389-396

13. Bindra A, Braunstein GD (2006) Thyroiditis. Am Fam Physician 73:1769-1776

14. Görges J, Ulrich J, Keck C, Müller-Wieland D, Diederich S, Janssen O (2019) Long-term outcome of subacute thyroiditis. Exp Clin Endocrinol Diabetes. https://doi.org/10.1055/a-0998-8035

15. Hennessey JV (2000) Subacute thyroiditis. In: Feingold KR, Anawalt B, Boyce A et al (Hrsg) Endotext. MDText.com, South Dartmouth (MA)

16. Lazarus JH (1996) Silent thyroiditis and subacute thyroiditis. In: Braverman LE, Utiger RD (Hrsg) The thyroid: a fundamental and clinical text. Lippincott Williams \& Wilkins, Philadelphia

17. Weihl AC, Daniels GH, Ridgway EC, MaloofF (1977) Thyroid function tests during the early phase 
of subacute thyroiditis. J Clin Endocrinol Metab 44:1107-1114

18. Chen X, Zhou Y, Zhou M, Yin Q, Wang S (2018) Diagnostic values of free triiodothyronine and free thyroxine and the ratio of free triiodothyronine and free thyroxine in thyrotoxicosis. Int J Endocrinol 2018:4836736

19. Pearce EN, Bogazzi F, Martino E, Brogioni $S$, Pardini $\mathrm{E}$, Pellegrini $\mathrm{G}$, Parkes $\mathrm{AB}$, Lazarus $\mathrm{JH}$, Pinchera A, Braverman LE (2003) The prevalence of elevated serum C-reactive protein levels in inflammatory and noninflammatory thyroid disease. Thyroid 13:643-648

20. Matsumoto Y, Amino N, Kubota S, Ikeda N, Morita S, Nishihara $E$, Ohye $H$, Kudo $T$, Ito $M$, Fukata $S$, Miyauchi A (2008) Serial changes in liver function tests in patients with subacute thyroiditis. Thyroid 18:815-816

21. Volpé R, Row VV, Ezrin C (1967) Circulating viral and thyroid antibodies in subacute thyroiditis. J Clin Endocrinol Metab 27:1275-1284

22. Frates MC, Marqusee E, Benson CB, Alexander EK (2013) Subacute granulomatous (de Quervain)thyroiditis: grayscale and color Doppler sonographic characteristics. JUltrasound Med 32:505-511

23. Lee YJ, Kim DW (2016) Sonographic characteristics and interval changes of subacute thyroiditis. JUltrasound Med 35:1653-1659

24. Park SY, Kim E-K, Kim MJ, Kim BM, Oh KK, Hong SW, Park CS (2006) Ultrasonographic characteristics of subacute granulomatous thyroiditis. Korean J Radiol 7:229-234

25. Lamichaney R, Sherpa M, Das D, Bhutia CT, Laishram S (2017) Fine-needle aspiration of de Quervain's thyroiditis (subacute granulomatous thyroiditis): a cytological review of 20 cases. J Clin Diagn Res 11:EC09:EC11

26. Stasiak M, Michalak R, Lewinski A (2019) Thyroid primaryand metastatic malignant tumours of poor prognosis may mimic subacute thyroiditis - time to change the diagnostic criteria: case reports and a review of the literature. BMCEndocr Disord 19:86

27. Sencar ME, Calapkulu M, Sakiz D, Hepsen S, Kus A, Akhanli P, Unsal IO, Kizilgul M, Ucan B, Ozbek M, Cakal $E$ (2019) An evaluation of the results of the steroid and non-steroidal anti-inflammatory drug treatments in subacute thyroiditis in relation to persistent hypothyroidism and recurrence. Sci Rep 9:16899

28. Sato J, Uchida T, Komiya K, Goto $H$, Takeno K Suzuki $R$, Honda A, Himuro $M$, Watada $H$ (2017) Comparison of the therapeutic effects of prednisolone and nonsteroidal anti-inflammatory drugs in patients with subacute thyroiditis. Endocrine 55:209-214

29. Benbassat CA, Olchovsky D, Tsvetov G, Shimon I (2007) Subacute thyroiditis: clinical characteristics and treatment outcome in fifty-six consecutive patients diagnosed between 1999 and 2005 . JEndocrinol Invest 30:631-635

30. Kubota $S$, Nishihara $E$, Kudo $T$, Ito $M$, Amino N, Miyauchi A (2013) Initial treatment with $15 \mathrm{mg}$ of prednisolone daily is sufficient for most patients with subacute thyroiditis in Japan. Thyroid 23:269-272

31. Mazza E, Quaglino F, Suriani A, Palestini N, Gottero C, Leli R, Taraglio S (2015) Thyroidectomy for painfulthyroiditisresistant to steroid treatment: three new cases with review of the literature. Case RepEndocrinol 2015:138327

32. Al-Dajani N, Wootton SH (2007) Cervical lymphadenitis, suppurative parotitis, thyroiditis, and infected cysts. Infect Dis Clin North Am 21:523-541
33. Kim BS, Nam KW, Kim JE, Park JH, Yoon JS, Park JH, Hong SM, Lee CB, Park YS, Choi WH, Ahn YH, Kim DS (2013) A case of acute suppurative thyroiditis with thyrotoxicosis in an elderly patient. Endocrinol Metab (Seoul) 28:50-54

34. Yu EH, Ko WC, Chuang YC, Wu TJ (1998) Suppurative Acinetobacter baumanii thyroiditis with bacteremic pneumonia: case report and review. Clin Infect Dis 27:1286-1290

35. Berger SA, Zonszein J, Villamena P, Mittman N (1983) Infectious diseases of the thyroid gland. Rev Infect Dis 5:108-122

36. Farwell AP, Braverman LE (1996) Inflammatory thyroid disorders. Otolaryngol Clin North Am 29:541-556

37. Herndon MD, Christie DB, Ayoub MM, Duggan AD (2007) Thyroid abscess: case report and review of the literature. Am Surg 73:725-728

38. Falhammar H, Wallin G, Calissendorff J (2019) Acute suppurative thyroiditis with thyroid abscess in adults: clinical presentation, treatment and outcomes. BMC Endocr Disord 19:130

39. Masuoka $\mathrm{H}$, Miyauchi $A$, Tomoda $\mathrm{C}$, Inoue $\mathrm{H}$, Takamura Y, Ito Y, Kobayashi K, Miya A (2011) Imaging studies in sixty patients with acute suppurative thyroiditis. Thyroid 21:1075-1080

40. Iwama S, Kato Y, Nakayama S (2007) Acute suppurative thyroiditis extending to descending necrotizing mediastinitis and pericarditis. Thyroid 17:281-282

41. Kawanaka M, Sugimoto Y, Suehiro M, Fukuchi M (1994) Thyroid imaging in a typical case of acute suppurative thyroiditis with abscess formation due to infection from a persistent thyreoglossal duct. Ann Nucl Med 8:159-162

42. Miyauchi A (2010) Thyroid gland: a new management algorithm for acute suppurative thyroiditis? NatRevEndocrinol 6:424-426

43. Chrobok V, Celakovský P, Nuńez-Fernández $D$ Simáková E (2000) Acute purulent thyroiditis with retropharyngeal and retrotracheal abscesses. JLaryngol Otol 114:151-153

44. Jacobs A, Gros DA, Gradon JD (2003) Thyroid abscess due to Acinetobacter calcoaceticus: case report and review of the causes of and current management strategies of thyroid abscesses. South Med J 96:300-307

45. Dugar M, da Graca Bandeira A, Bruns J Jr, Som PM (2009) Unilateral hypopharyngitis, cellulitis, and a multinodular goiter: a triad of findings suggestive of acute suppurative thyroiditis. AJNR Am J Neuroradiol 30:1944-1946

Hinweis des Verlags. Der Verlag bleibt in Hinblick auf geografische Zuordnungen und Gebietsbezeichnungen in veröffentlichten Karten und Institutsadressen neutral.

\section{Bewegung schützt vor Diabetes - aber wie?}

Typ-2-Diabetes kündigt sich meist an: Die Patienten haben einen dauerhaft erhöhten Blutzuckerwert ohne jedoch die für die Diabetesdiagnose festgelegten Grenzwerte zu überschreiten. In diesem Fall handelt es sich um Prädiabetes. Menschen mit Prädiabetes erhalten die ärztliche Empfehlung, regelmäßig Sport zu treiben. Häufig lässt sich die Diabeteserkrankung durch Bewegung verhindern. Ein bestimmter Anteil der Betroffenen weist jedoch eine Trainingsresistenz auf: Bei innen zeigt der Sport keinerlei Wirkung oder fördert gar die Entwicklung von Diabetes. Gianni Panagiotou vom Leibniz-Institut für Naturstoff-Forschung und Infektionsbiologie in Jena und seine Kollegen von der Universität Hongkong haben das Darmmikrobiom von Patienten, die positiv auf Sport ansprechen mit dem von jenen verglichen, wo Bewegung keine Wirkung zeigt. Die Studiendaten zeigen einen klaren $\mathrm{Zu}$ sammenhang zwischen Darmmikrobiom, Bewegung und Blutzuckerwerten. „Es gibt eine hohe Variabilität bei der Reaktion von Blutzuckerwerten auf sportliche Betätigung. Wir konnten herausfinden, dass diese in Abhängigkeit des Darmmikrobioms steht", sagt Panagiotou. Dabei beziehen sich diese Unterschiede sowohl auf die Zusammensetzung des Mikrobioms als auch auf seine Funktionalität. So produziert das Darmmikrobiom von erfolgreich Therapierten mehr nützliche kurzkettige Fettsäuren während bei denen, die nicht ansprachen, eher metabolisch schädliche Verbindungen auftreten. Die Ergebnisse wurden anhand des Darmmikrobioms von 39 Männern mit Prädiabetes gewonnen. In einem Kontrollexperiment wurde das Darmmikrobiom der unterschiedlich reagierenden Patienten aus dieser Gruppe auf fettleibige Mäuse übertragen. Allein das Mikrobiom derer, bei denen die Bewegungstherapie wirksam war, führte bei den Mäusen zu denselben positiven Auswirkungen. Mit der Untersuchung des Darmmikrobioms ließe sich also voraussagen, wie gut Prädiabetespatienten auf Bewegung ansprechen.

Quelle: www.leibniz-hki.de 